\title{
Critical-Sized Bone Defect Regeneration: A Novel Scaffold Made by Electrospinning of Metformin-Incorporated Gela- tin/Hydroxyapatite Nano-Fibers
}

\author{
Chung-Kai Sun ${ }^{1,+}$, Pei-Wei Weng ${ }^{2,+}$, Jenny Zwei-Chieng Chang ${ }^{3}$, Yi-Wen Lin ${ }^{4}$, Fon-Yih Tsuang 5, Feng-Huei Lin \\ ${ }^{6}$, Tung-Hu Tsai ${ }^{7, *}$ and Jui-Sheng Sun ${ }^{8, *}$
}

1 Institute of Traditional Medicine, School of Medicine, National Yang Ming Chiao Tung University, No.155, Sec.2, Linong Street, Taipei 11221, Taiwan, ROC; samcksun@gmail.com

2 Department of Orthopaedics, Taipei Medical University-Shuang Ho Hospital, Ministry of Health and Welfare, No.291, Zhongzheng Rd., Zhonghe District, New Taipei City, 23561, Taiwan, ROC; wpwort@gmail.com

3 School of Dentistry, College of Medicine, National Taiwan University, No 1, Chang-Te Street, Taipei 10048, Taiwan, ROC; jennyzc@ms3.hinet.net

4 Institute of Biomedical Engineering, College of Medicine and College of Engineering, National Taiwan University, No. 1, Sec. 4, Roosevelt Rd, Taipei 10617, Taiwan; zhew520@gmail.com

5 Division of Neurosurgery, Department of Surgery, National Taiwan University Hospital, No. 7, ChungShan South Road, Taipei 10035, Taiwan, ROC; tsuangfy@gmail.com

6 Institute of Biomedical Engineering, College of Medicine and College of Engineering, National Taiwan University, No. 1, Sec. 4, Roosevelt Rd, Taipei 10617, Taiwan; double@ntu.edu.tw

7 Institute of Traditional Medicine, School of Medicine, National Yang Ming Chiao Tung University, No.155, Sec.2, Linong Street, Taipei 11221, Taiwan, ROC; thtsai@ym.edu.tw

8 Department of Orthopedic Surgery, College of Medicine, China Medical University, No. 2, Yu-Der Rd, Taichung City 40447, Taiwan, ROC; and Department of Orthopedic Surgery, National Taiwan University Hospital, No. 7, Chung-Shan South Road, Taipei 10035, Taiwan, ROC; drjssun@gmail.com

+ Both Pei-Wei Weng and Chung-Kai Sun contribute equally as the co-first authors.

* Correspondence: T-H Tsai: thtsai@ym.edu.tw and J-S Sun: drjssun@gmail.com;

\begin{abstract}
Tissue engineering and regenerative medicine has gradually evolved as a promising therapeutic strategy to the modern healthcare of the aging and diseased population. In this study, we developed a novel nano-fibrous scaffold and verified its application in the critical bone defect regeneration. The metformin-incorporated nano-gelatin/hydroxyapatite fibers (NGF) was produced by electrospinning, cross-linked, and then characterized by XRD and FTIR. Cytotoxicity, cells adhesion, cell differentiation, and quantitative osteogenic gene and protein expression were analyzed by bone marrow stem cells from rat. Rat forearm critical bone defect model was performed for the in vivo study. The nano-gelatin/hydroxyapatite fibers (NGF) were characterized by their porous structures with proper interconnectivity without significant cytotoxic effects; the adhesion of bone marrow stem cells on the nano-gelatin/hydroxyapatite fibers (NGF) could be enhanced. The osteogenic gene and protein expression were upregulated. Post implantation, the new regenerated bone in bone defect was well demonstrated in the NGF samples. We demonstrated that the metformin-incorporated nano-gelatin-hydroxyapatite fibers greatly improved healing potential on the critical sized bone defect. Although metformin-incorporated nano-gelatin/hydroxyapatite fibers had advantageous effectiveness during bone regeneration, further validation is required before it can be applied to clinical applications.
\end{abstract}

Keywords: critical sized bone defect; bone tissue regeneration; nano-gelatin/ hydroxyapatite fiber (NGF); metformin.

\section{Introduction}

Bone is the structure that supports the rest of the body, acts as levers for muscles to allow for locomotion and movement, protects internal organs and structures; recent study 
have shown that bone provides hematopoiesis of human body, maintains acid-base balance and homeostasis of mineral, also acts as a place for storing growth factors and cytokines [1].

Like all tissues, the extracellular matrix is produced and secreted by different cells of the bone tissue to provide biophysical and biochemical support to nearby cells [2]. Within the bone matrix, the most abundant protein is type I collagen [2, 3]; while at different stages of bone formation, trace amounts of calcium-phosphate binding proteins, such as alkaline phosphatase, osteopontin, osteocalcin, and bone sialoprotein can be found to help regulate the hydroxyapatite crystal formation and mineral deposition in an ordered fashion [4]. The mechanical rigidity and load-bearing strength of bones is mainly come by minerals within the bones, while the organic matrix provides the elasticity and flexibility of the bone [5]. Both components are vital for the overall function and health of the bones.

Tissue engineering and regenerative medicine has gradually evolved as a promising therapeutic strategy to the modern healthcare of the aging and diseased population. Biomaterials are developed in the aim of healing, repairing or regenerating damaged tissues and organs [6]. Recent studies have demonstrated that extracellular matrix scaffolds create microenvironment favorable for tissue regeneration. They are able to act as a pattern for different tissues' repair and promote tissue-specific remodeling [2]; various studies have demonstrated electrospinning as the promising method for manufacturing scaffold for use in bone tissue engineering [7, 8]. Different materials have been tested as materials for scaffolds, such as chitosan/collagen [9], polycaprolactone [10], carboxymethyl chitosan [11], and gelatin [12]. Among these, gelatin is considered to have good cell adhesion, proliferation and promoting bone formation [7].

Collagen, the main extracellular matrix protein of bone tissues, contains the tripeptide Arg-Gly-Asp (tripeptide RGD) motif to facilitate cell attachment [2,3]. Ais a protein derived from hydrolysis of collagen and mimicked natural extracellular matrix, scientists frequently use gelatin as the scaffold in the modern tissue engineering [13]. Nevertheless, the application of gelatin is limited by the enzymatic degradation in vivo and lack of mechanical strength. In general, collagen-based biomaterials require the introduction of additional support and/or cross-linkage to achieve adequate performance in vivo. As mechanical strength is mandatory for the bone tissue engineering, hydroxyapatite is a good ingredient to provide additional strength for scaffolds [14, 15]. Regarding cross-linkers, chemicals such as glutaraldehyde, formaldehyde, and diphenyl-phosphoryl azide (DPPA) are most commonly utilized. In this study, a novel cross-linked gelatin/ hydroxyapatite nano-fibers (NGF) was developed by electrospinning to form scaffold, and its application in the regeneration of bone defect with critical size was verified.

\section{Results}

\subsection{Morphology of Nano-Gelatin/ Hydroxyapatite fibers (NGF)}

The morphology of nano-gelatin/hydroxyapatite fibers (NGF) were characterized by using SEM, and the surface morphology and fiber diameter were analyzed (Figure 1B). The gelatin nanofibers showed smooth surface with a diameter of $100 \pm 10 \mathrm{~nm}$ and porous structures with proper interconnectivity were seen. 


\section{A. Preparation of nanofibers}

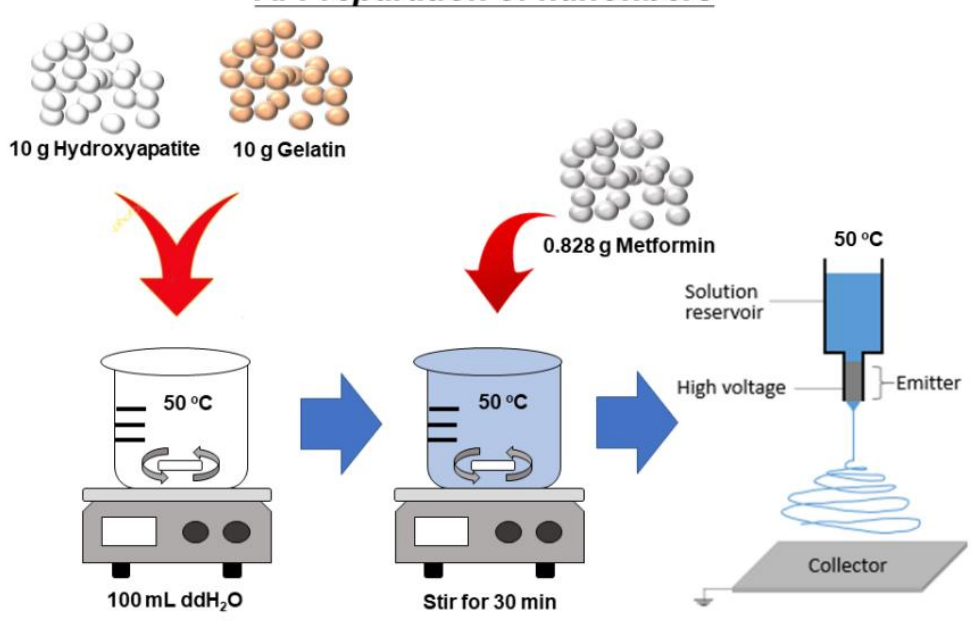

B. Morphology of gelatin/hydroxyapatite nanofibers

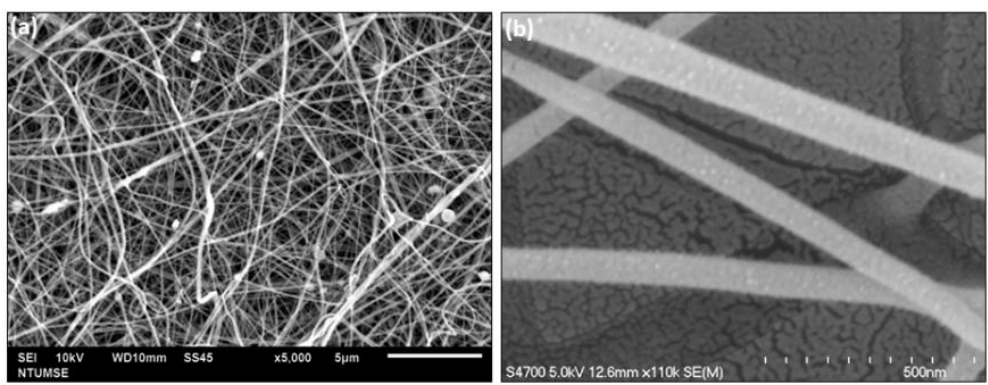

Figure 1. The preparation and morphology of metformin-incorporated gelatin/ hydroxyapatite nanofibrous Scaffold. 1A. The preparation of metformin-incorporated gelatin/ hydroxyapatite nanofibrous Scaffold. 1B. Microscopic images of nano-gelatin/ hydroxyapatite fibers (NGF). (a) magnification: $x 5 \mathrm{k}$; (b) magnification: $x 110 \mathrm{k}$. The fiber diameter and surface morphology of the gelatin nanofibers were characterized and analyzed by SEM. The gelatin nanofibers showed smooth surface with a diameter of $100 \pm 10 \mathrm{~nm}$ and porous structures with proper interconnectivity were seen.

\subsection{Characterization of Nano-Gelatin/ Hydroxyapatite fibers (NGF)}

Over $2 \theta$ range from $10^{\circ}$ to $70^{\circ}$, the pattern of XRD was collected with an incremental step size of $0.04^{\circ}$, which indicated the crystal phase of NGF as showed in figure 2A. The XRD pattern of nano-gelatin/ hydroxyapatite fibers (NGF) scaffolds exhibited sharp diffraction peaks similar to that of the hydroxyapatite. The peaks of both natural bone and NGF match well, suggesting that NGF's similarity to the bone tissue (Figure 2A). The FTIR pattern showed the functional group of NGF in figure $\mathbf{2 B}$, the peak of amide, phosphate, hydroxide, and the carbonate group also matched the natural bone, suggesting that the characteristic of NGF was quite similar to the natural bone (Figure 2B). 


\section{Characterization of Nano-Gelatin/Hydroxyapatite fibers nanofibers}

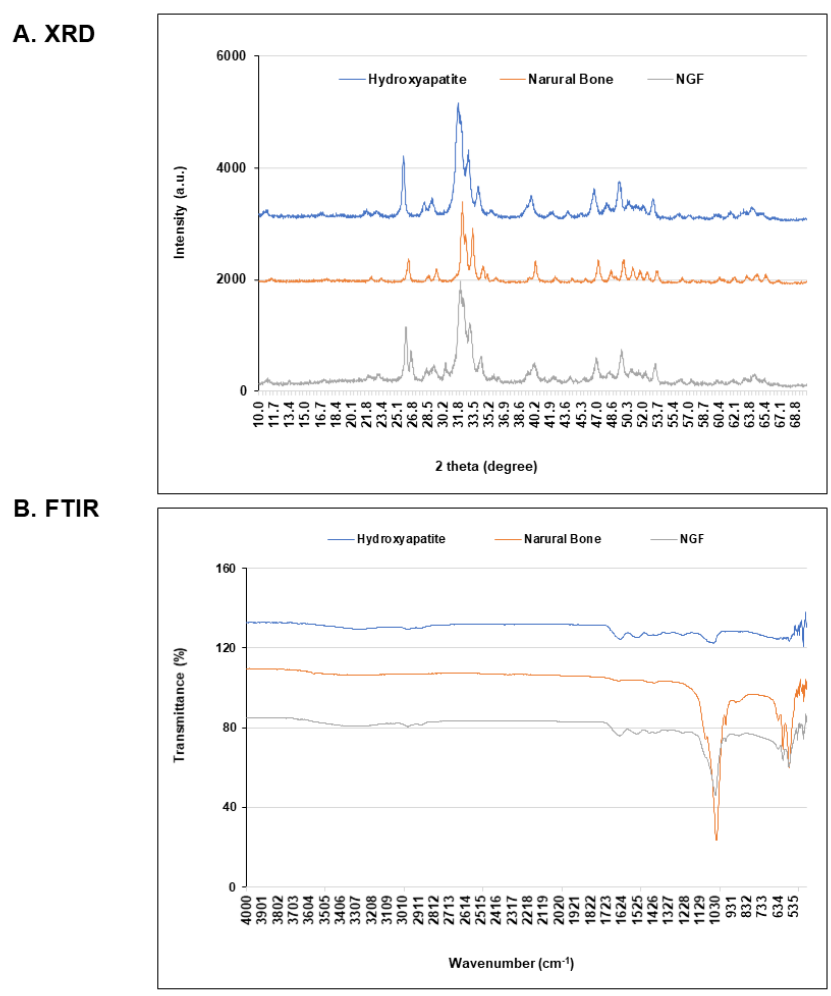

Figure 2. Characterization of nano-gelatin/ hydroxyapatite fibers by XRD and FTIR. 2A. Over a $2 \theta$ range from $10^{\circ}$ to $70^{\circ}$, the pattern of XRD was collected with an incremental step size of $0.04^{\circ}$, and that indicated crystal phase of gelatin/ hydroxyapatite nano-fibers (NGF). The XRD pattern of nano-gelatin/ hydroxyapatite fibers (NGF) scaffolds exhibited sharp diffraction peaks which was similar to the hydroxyapatite. Both peaks of natural bone and NGF match well, suggesting that NGF is similar to the bone tissue. 2B. The FTIR pattern showed the functional group of NGF, the peak of amide, phosphate, hydroxide, and the carbonate group, also match to the natural bone, suggesting that NGF was similar to the natural bone.

\subsection{Cytotoxicity and Cells Adhesion of Nano-Gelatin/ Hydroxyapatite fibers (NGF)}

Cytotoxicity and cells adhesion behavior on the nano-gelatin/ hydroxyapatite fibrous scaffolds (NGF) were examined (Figure 3). The cytotoxicity of NGF was assessed by WST-1 assay and live/death test. As shown at figure 3, NGF did not possess any significant cytotoxic effects on cells within 1 and 3 days (Figure 3A). When assessed by the live/death staining assay, both the cells of control and NGF groups showed good cell viability at the day 3 in cytotoxicity and cell adhesion study of gelatin/ hydroxyapatite nanofibrous scaffold (Figure 3B). After phalloidin staining of the F-actin, the cytoskeleton organization and cellular morphology on the constructs were analyzed. When comparing to the Petri dish as control and NGF (Figure 3C), NGF could enhance the formation of F-actin for cells adhesion and which appeared as diffuse actin staining inside the cells. 


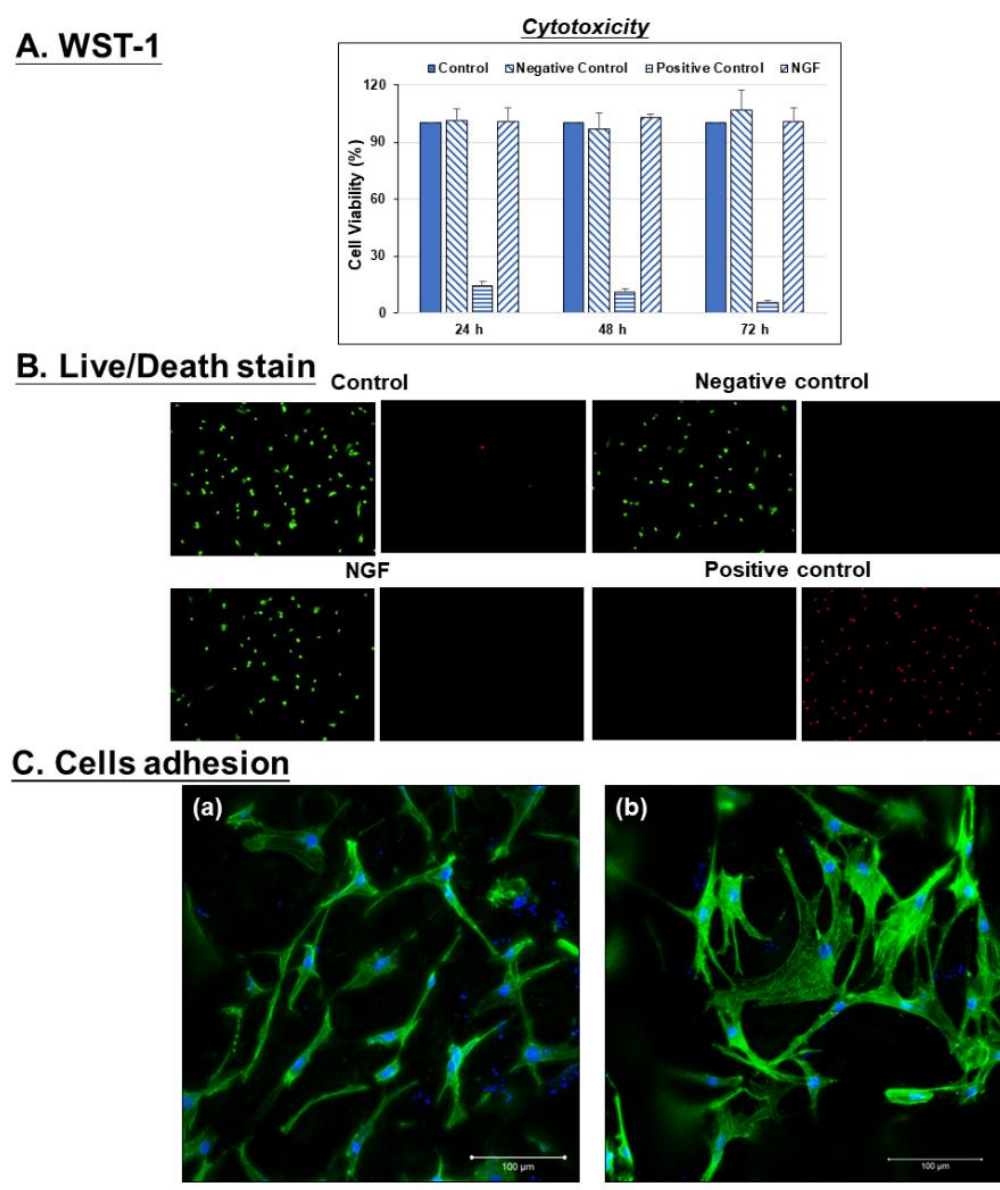

Figure 3. Cytotoxicity and cell adhesion study of gelatin/ hydroxyapatite nanofibrous scaffold. 3A. The cytotoxicity of NGF was assessed by the WST-1 assay. Control group was $100 \%$ cell viability, negative control was aluminum oxide, and positive control was zinc diethyldithiocarbamate. The cells have been seeded on the NGF and maintained in culture for three days. As shown, no significant cytotoxic effects on cells at either 1 or 3 days of culture. 3B. Cytotoxicity of the NGF was assessed by the live/death staining assay. The cells of both the control and NGF groups showed good cell viability at the day 3 , indicating there were no significant cytotoxic effects on cells. $3 \mathrm{C}$. Cell adhesion on the gelatin/ hydroxyapatite nanofibrous scaffold. After phalloidin staining of the Factin, the cytoskeleton organization and cellular morphology and of the cells on the constructs were analyzed. When comparing to the cells grown in Petri dish as control (a) and on NGF (b), enhanced expression of F-actin was remarked inside the cells in contact with NGF suggesting a better cell adhesion.

\subsection{Osteogenesis-related Gene Expression}

A cascade of changes in intracellular genes or proteins expression [including alkaline phosphatase (ALP), runt-related transcription factor-2 (RUNX-2), osterix (SP7), osteocalcin (BGLAP), osteonectin (SPARC) and collagen type I (COL1A1)] regulated the osteogenic differentiation BMSCs. The interactions between cells and extracellular matrixes could be revealed by transcript levels of these osteogenesis-related genes and proteins. When BMSCs cultured with NGF for four weeks, qRT-PCR assays were carried out to analyze osteo-specific gene expression. As expected, the osteogenic specific genes, including ALP, Runx2, SP7, BGLAP, SPARC, and Col1A1 were upregulated when normalized to the control group (Figure 4A).

\subsection{Expression of Osteogenic-Specific Proteins}

The expression of osteogenic-specific proteins, including alkaline phosphatase (ALP), osteonectin $(\mathrm{ON})$, osteocalcin $(\mathrm{OC})$, and type I collagen (COL1A1), were analyzed with 
ELISA kits. At four weeks, the osteogenic specific proteins were all upregulated when comparing to that of the control group. As shown, the ALP expression upregulated in earlier stage, OC and ON expression in medium increased to a later stage, and COL1A1 expression al so in later stage (Figure 4B). Similar results were also demonstrated in the immunofluorescence staining of osteogenic differentiation (Figure 4C).

\section{A. Gene expression of osteogenic differentiation}

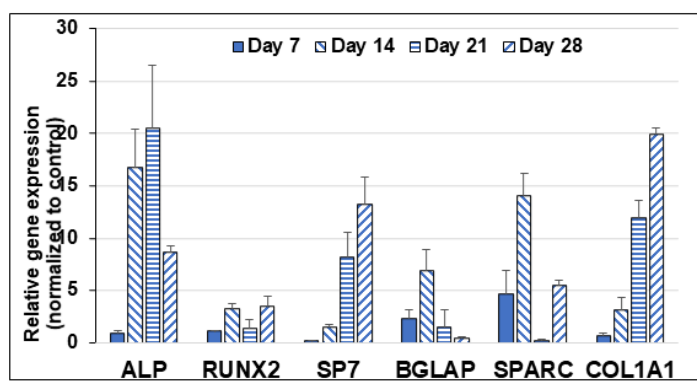

\section{Immunofluorescence staining}
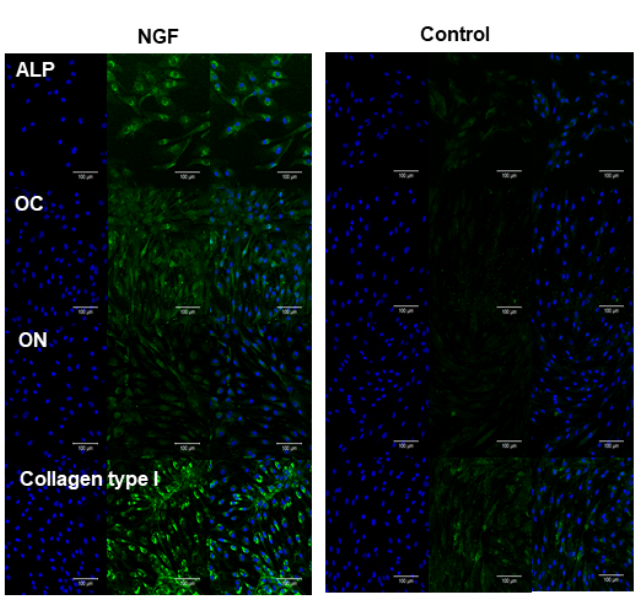

\section{B. Protein expression of osteogenic differentiation}
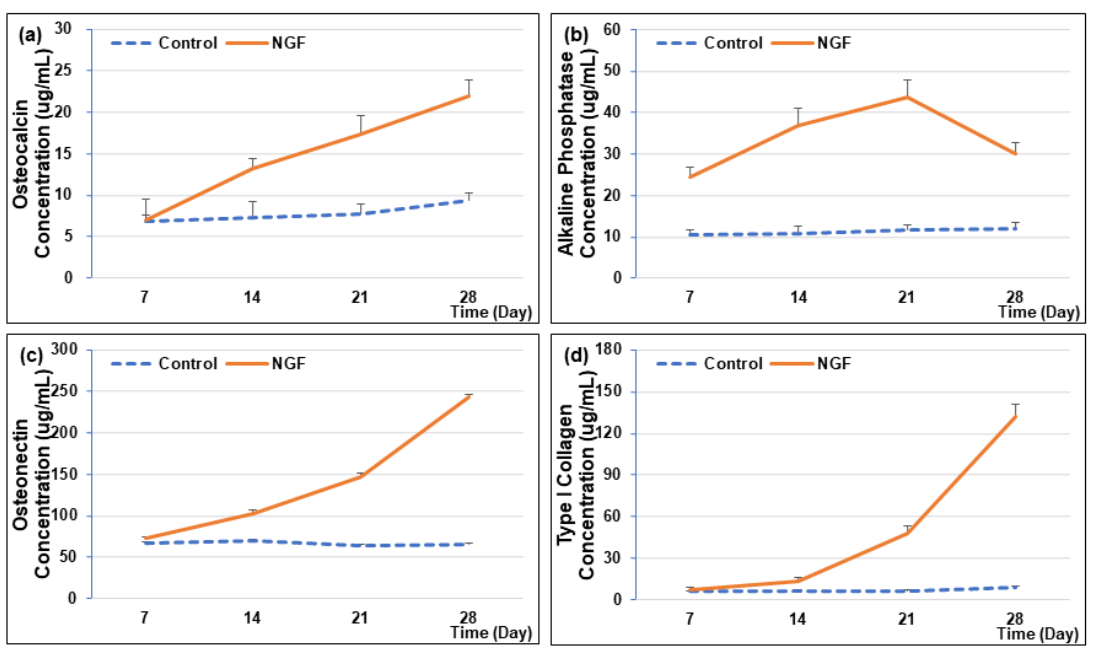

Figure 4. Gene/ protein expression and immunofluorescence staining of osteogenic markers. 4A. Gene expression of osteogenic markers. The osteoblast specific genes of MSCs, including alkaline phosphatase (ALP), runt-related transcription factor-2 (RUNX-2), osterix (SP7), osteocalcin

(BGLAP), osteonectin (SPARC), and type I collagen (COL1A1) were upregulated when normalized to the control group. 4B. Expression of osteogenic proteins. ELISA kits were used to analyze osteogenic-specific protein expression from BMSCs cultured on NGF. Protein markers of osteogenesis of BMSCs, including ALP, ON, OC, and collagen type I were all upregulated when comparing to that of the control group. (a) alkaline phosphatase; (b) osteocalcin; (c) osteonectin; (d) collagen type I in $7,14,21$, and 28 days. As shown, the ALP over expression in early stage, OC and ON over expression in medium to later stage, and COL1A1 expression in later stage. 4C. Immunofluorescence staining of alkaline phosphatase (ALP), osteocalcin (OC), osteonectin $(\mathrm{ON})$, and collagen type I after 28 days.

\subsection{In Vivo Post-Implantation New Bone Formation}

New bone formation in control, Sinbone, and NGF samples were observed by micro-CT scan examination at 12 weeks post-implantation. As shown, bone defect at the osteotomy site of was still quite evident in the control and there was a substantial amount of implant 
granules remained in the defect site when the defects were treated with Sinbone samples; while, well-developed bone regeneration was found in the NGF sample (Figure 5A). At 1 month post operation, the NGF group showed the most prominent regeneration and which continued to improve over the three month period. A well-developed new bone formation was found in the NGF samples, the bone volume density at the defect site was significantly higher than that of control and SinBone treated samples (Figure 5B).

\section{A. Micro computed tomography}

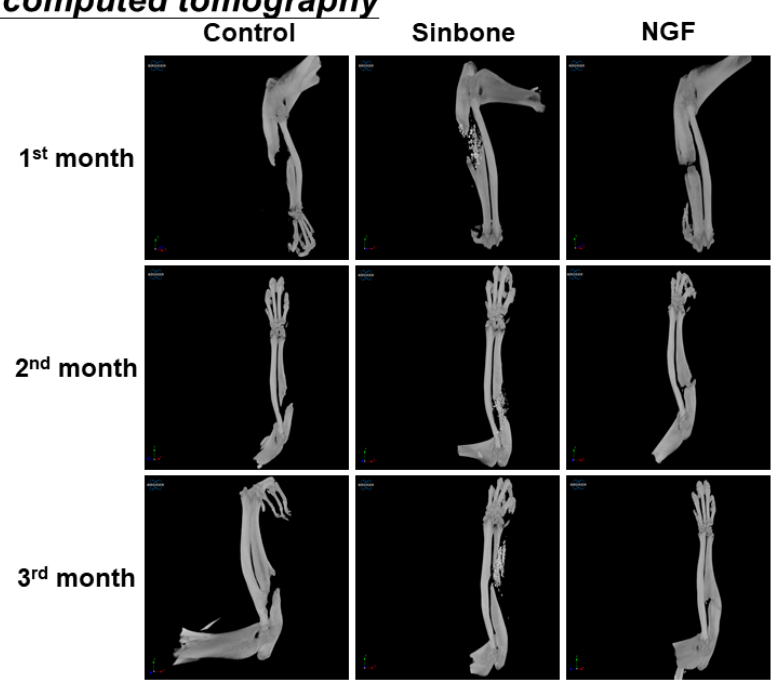

\section{B. Bone volume density (BV/TV)}

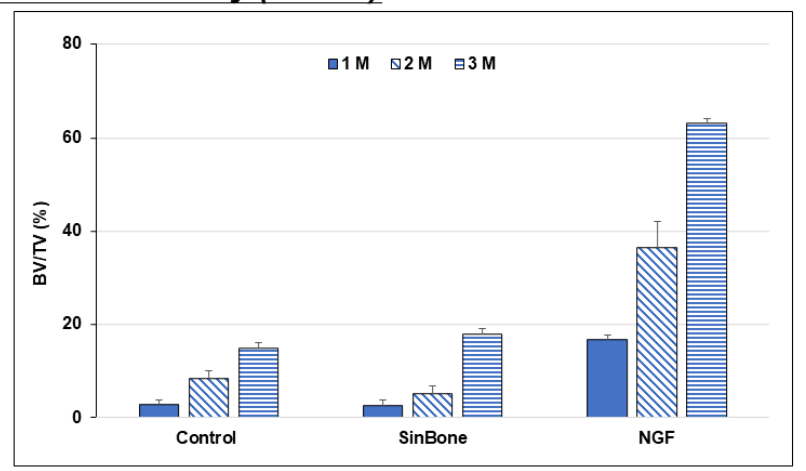

Figure 5. Post-implantation new bone formation: Micro computed tomography. 5A. Micro-Computed Tomography comparison of control, Sinbone and NGF at 1 month, 2 months, and 3 months postoperative day respectively. At 12 weeks post-implantation, new bone formation in the control, Sinbone, and NGF samples were observed by micro-CT scan examination. Bone defect at the osteotomy site of was still quite evident in the control. A substantial amount of ganules remained in the defect site when the defects were treated with Sinbone samples. While, a well-developed new bone formation was found in the NGF samples. Comparatively, the NGF group showed the most prominent regeneration even at 1 months post operation, and continued to improve over the three month period. 5B. Micro-CT scan. At 12 weeks post-implantation, new bone formation in the control, Sinbone, and NGF samples were observed by micro-computed tomography examination. A well-developed new bone regeneration was found in the NGF sample, the bone volume density at the defect site was significantly higher than that of control and SinBone treated samples.

In the control samples, the defect site still filled with invading fibrous connective tissue with little new bone formation even at 12 weeks post-implantation. In those bone defects treated with Sinbone granules, bone regeneration was shown only within the pores of hydroxyapatite particles and that a substantial amount of Sinbone granules still remained within the defect site. While, a well-developed new bone regeneration was found in the NGF sample (Figure 6; H\&E stain). The Goldner's trichrome staining showed minimal new bone formation in the control samples, whereas immature new bone formation with soft 
tissue invasion observed in the Sinbone-treated samples; while well-developed new bone and hyaline cartilage (endochondral bone formation) was found in the NGF samples (Figure 6; Goldner's trichrome stain).

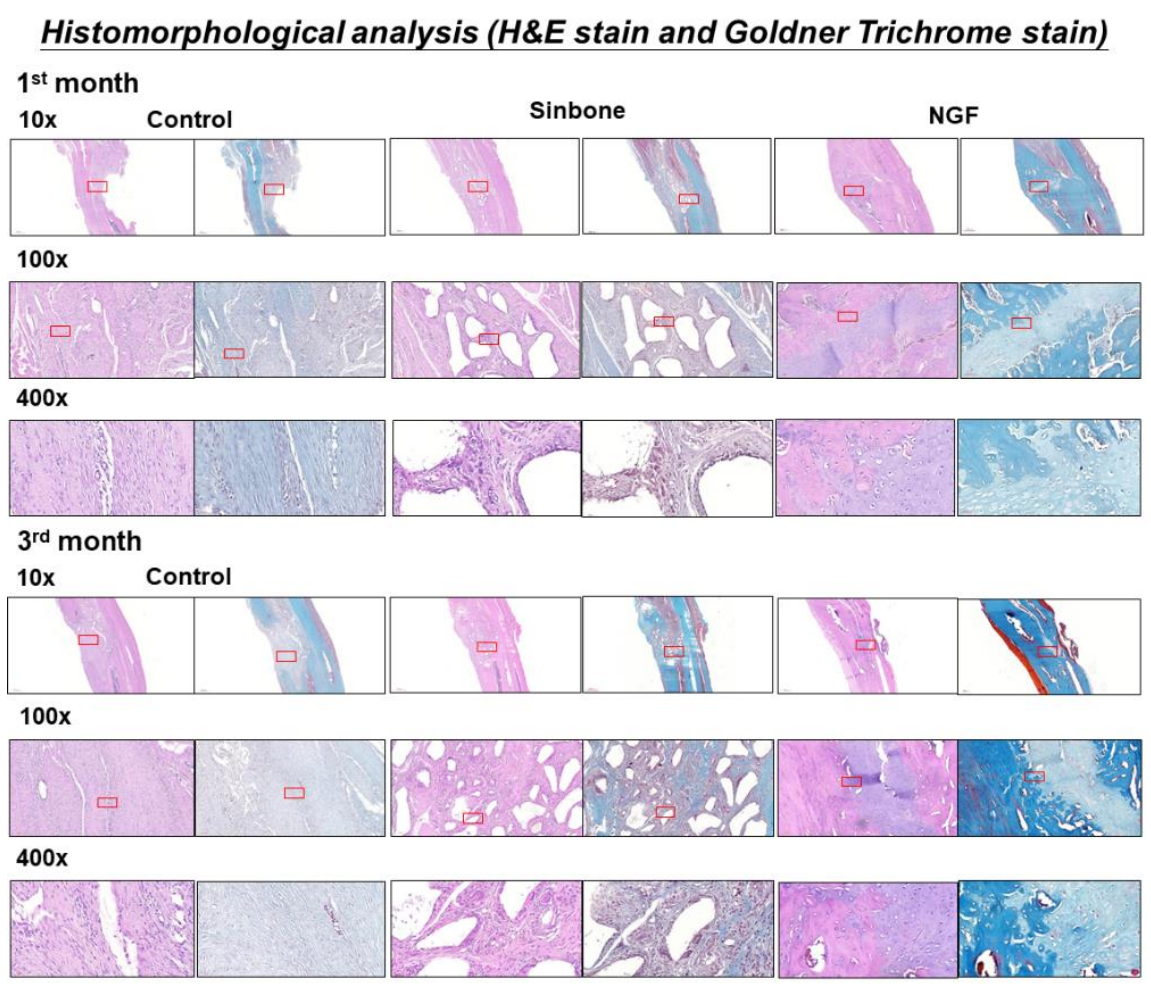

Figure 6. Post-implantation new bone formation: Histomorphological analysis.

Hematoxylin \& eosin (H\&E) staining. At 12 weeks post-implantation, new bone formation in the control, Sinbone, and NGF samples were observed by H\&E staining. New bone growth was not evident in the control sample, with the defect site filled with invading fibrous tissue. The defects treated with Sinbone granules showed new bone growth only within the pores of hydroxyapatite particles with a substantial amount of implant still remained in the defect site. While, a well-developed new bone regeneration was found in the NGF sample.

Goldner's Trichrome staining. At 12 weeks post-implantation, new bone formation in the control, Sinbone, and NGF samples were observed by Goldner's trichrome staining. Goldner's trichrome staining shows a minimal new bone formation in control samples, whereas immature bone formation with fibrous tissue invasion were observed in Sinbone samples. A well-developed new bone and hyaline cartilage (endochondral bone formation) was found in the NGF samples. 


\section{Discussion}

A successful bone graft substitute should be non-toxic, non-antigenic, biocompatible, bioactive, biodegradable, and microporous to provide angiogenesis and new bone ingrowth; it should also be easily operable [16]. Autografts, allografts, xenografts, ceramics, polymers and even metals are the commonly used bone graft substitutes to promote bone reunion $[16,17]$. With similar structure as the native extra cellular matrix $(E C M)$, some biodegradable natural polymers (such as collagen, gelatin, fibrin, hyaluronic acid, etc.) they have been widely applied in the design of scaffolds for tissue engineering $[16,18,19]$. In this study, we used a novel developed electrospinning nanogelatin/hydroxyapatite fibrous (NGF) scaffolds to verify its application in bone regeneration.

BMSCs are easy to isolate and readily expandable in vitro and are one of the major stem cells sources in bone tissue engineering. In this study, the synthesized nano-gelatin/ hydroxyapatite fibrous scaffold possessed smooth surface and porous interconnectivity, which showed good cell viability and could enhance the cells adhesion. Relative to the control group, the osteoblast specific genes, including ALP, Runx2, SP7, BGLAP, SPARC, and Col1A1 were all upregulated. The expression of Runx2 gene showed 3.2-fold to 3.4fold upregulation. Runx2, a master regulator of bone development, is essential for the expression of a number of bone-specific genes and plays an important role in regulating bone development and osteoblastic function [20]. Moreover, Runx2 expression can upregulate the bone matrix genes (BGLAP, Col1a1, etc.) expression as well as regulate the differentiation of BMSCs into osteoblasts [21]. On the process of osteogenic differentiation, BGLAP is the most specific gene expression for regulation of osteoblast differentiation and mineralization [22]. In this study, when BMSCs were cultivated on the NGF, the expression of Col1a1 significantly increased on day 7 and 14; BGLAP gene expression showed the higher increase (6.9-fold) when BMSCs were cultured on NGF for 14 days.

Type I collagen (Col1A1) is the most abundant protein of the extracellular bone matrix and has an important role in influencing bone cells behavior [23]. The osteogenic differentiation of BMSCs in vitro can be divided into three different stages [24]. In the first stage (days one to four), active cellular proliferation is seen. Followed by the second stage of early cell differentiation (from days 5 to 14), which is characterized by the alkaline phosphatase (ALP) gene transcription and protein expression [25]. After its initial peak, the ALP's level declines and the type I collagen expression is also found at this stage [26]. At the third stage (from days 14 to 28), high osteocalcin and osteopontin expression is followed by calcium and phosphate deposition [24, 27]. ALP is the most commonly used early marker of osteoblast differentiation. In this study, ALP protein experienced over expression in early stage, $\mathrm{OC}$ and $\mathrm{ON}$ protein during medium to later stage, and COL protein primarily in the later stage.

With its superior biocompatibility, hydroxyapatite (HA) is widely applied in the field of bone tissue repair. As the processing parameters often differ among different research groups, the properties of the end material are usually dependently changed. For such circumstance, two types of porous HA ceramics prepared by two different research teams, may be identical in their chemical composition, but totally differ in their osteoinductive potential as they are fully different in their grain size, surface roughness and macroporosity $[28,29]$. Until now, we are still unable to describe how an optimal osteoinductive material should be designed and produced. A recent study showed that HA nanoparticles (n-HA)-releasing scaffold can promote bone regeneration. This finding provides a rationale for using n-HA in the repair of segmental bone defects [30] and possible potential in bone tissue engineering. In this study, BMSCs could be differentiated to osteoblast lineage which was consider to be the nano-size effect of NGF.

The radial bone defect model of rat has previously been reported to be highly suitable for study of new bone regenerative materials, since it does not require any 
fixation that can influences the overall healing process [16]. In this study, the 5-mm length segmental defect was created in the mid-portion of the bone to prevent spontaneous healing and to set up a nonunion model with critical size bone defect [31]. Based on the previous in vitro reports, gelatin composites have been suggested for promoting bone repairs, and it can be suggested as a suitable material for scaffold fabrication in this study [32]. However, good in vitro cell viability and differentiation cannot guarantee the good in vivo role of gelatin [16]. In this study, the bone healing potential of NGF, Sinbone and their sham-operated control group in the critical bone defect model of rat were further evaluated. Our in vivo findings are in agreement with previously mentioned in vitro results. For the empty defect control group, the fibrous connective tissue was the main constituent within the defects during the whole healing process; while the defect treated with Sinbone alone was associated with minor healing potential superior to that of the control group. Here, we showed that NFG has excellent potential for in vivo bone regeneration and that its potential in promoting bone healing was superior to Sinbone and also superior to the previously reported for the in vivo role of gelatin [16].

It is a complicated issue when studying the healing process of critical size defects on weight bearing bones. In a previous study on the healing of critical sized bone defects, Oryan et al. (2016) concluded that chitosan-gelatin scaffold was inferior to the gelatin scaffold alone [16]. In this study, we experimentally created an ulnar bone defect in rat which was filled the NGF scaffold. The difference between our results and those of Oryan et al. may be due to the difference in protocol for preparation of gelatin scaffold, there was higher concentration of gelatin scaffold in our study when compared with those of Oryan et al. (10\% vs. $4.29 \mathrm{wt} \%$ ) [16]. This improvement might also be attributed to the higher bioactivity properties of the nano-sized electro-spun gelatin fibers used in our study. The present experiment showed that nano-gelatin fibers might have a positive effect in bone regeneration [33].

The specific surface area and their associated adsorption capacity of a material will be strongly increased as the particle size decreased. When compared to the commonly used moisture-sorbent silica gel, the amount of water adsorbed in these nano-sized HA particles was surprisingly high [34]. Towards biofabrication-based tissue repair, the nanometer dimensions inspired their biological potential; when compared to metallic implants coated with conventional or micron-sized HA, nanoparticles can promote osteoblast attachment and enhance other cell functions. In vitro and in vivo studies have shown that metallic implants coated with nano-hydroxyapatite (HA) promotes healing potential and reduces the time for complete osseointegration [35]. Osteo-conductivity of a biomaterial is the ability to guide new bone formation and establishes the bone continuity; on the other hand, osteo-inductivity is the ability to regenerate a new bony matrix by progenitor cells and synthesis of new bone matrix [16]. As the biocompatible biomaterials downgraded to much smaller nano-size scale, nano-hydroxyapatite and gelatin nanofiber might manifested osteoinductive by the adsorption of water and subsequent growth factors and cytokines in the surrounding tissue.

As a blood derivative, fibrin-platelet glue (FPG) can progressively release myriad of growth factors and proteins to the local environment from its biocompatible 3dimensional fibrin scaffold to accelerate postoperative bone healing. In a study of the critical bone defect of rats, Gholipour et al. concluded that the FPG improved the effectiveness of gelatin [33]. In our study of the experimental critical bone defect, the FPG was replaced by metformin-incorporated NGF; and even more important, all our major components (gelatin and hydroxyapatite) are nano-sized in scale.

As a second-generation biguanide used oral antidiabetic drug, metformin (MF) has been demonstrated to stimulate osteoblasts and reduce alveolar bone loss. Metformin was also found to increase bone density in diabetic animals and be protective to the bone loss [36]. The skeleton may also have an endocrine role. As an energy metabolism sensor, 
the AMP-activated protein kinase (AMPK) can regulate BMSC differentiation, stimulate osteogenesis and inhibit adipogenesis through the AMPK-Gfi1-OPN axis [37]. The AMPK also plays an important role on osteoblasts in glucose metabolism and in maintaining trabecular bone volume, cortical thickness, and then the bone strength [38]. Our study suggests that metformin may promote osteogenic differentiation and beneficial for bone formation [39]; therefore, metformin might be beneficial for the healing of criticalsized bone defect by acting on mature osteocytes. Recently, our study in large animal model using LanYu-100 minipig model (Affidavit of Approval of Animal Use Protocol, IACUC of Pigmodel Animal Technology Co., Ltd.: Approval No. PIG-108026) also demonstrated promising results with good bony incorporation of metformin-incorporated nano-gelatin/ hydroxyapatite fibrous scaffold (Figure Supplementary). Although the animal models may closely represent the situation of the human clinical situation, the anatomic and physiologic variations do exist between human beings and each animal model and this still need to be considered when the results of our study will be translated into clinical medicine.

\section{Materials and Methods}

\subsection{Electrospinning}

The homogeneous solution of 10 gm gelatin (Type A, porcine, Sigma-Aldrich, USA)/ 10 gm hydroxyapatite ( $<200 \mathrm{~nm}$ in particle size, Sigma-Aldrich, USA) and 0.828 gm of metformin (Metformin $\mathrm{HCl}$, M.W. 165.62, Sigma-Aldrich, USA) in $100 \mathrm{~mL}$ dd- $\mathrm{H}_{2} \mathrm{O}$ was prepared (at $50^{\circ} \mathrm{C}$ ) by magnetically stirred for 30 minutes (Figure 1A). The homogeneous solution was filled in $10 \mathrm{~mL}$ syringe with the temperature inside the syringe controlled at $45-70^{\circ} \mathrm{C}$ by a heater (AREX-6, VELP Scientifica, USA) within the syringe holder. The connected needle (inner diameter $1.5 \mathrm{~mm}$ ) applied $20 \mathrm{kV}$ potential to droplets of the injected solution which was generated by a high-voltage power supply (SC-PME50, Cosmi Global Co., Ltd., Taiwan). The aluminum foil-covered collector was set at a 5-cm distance from the capillary tip. The electro-spun fiber mats were collected and left for at least 3-h to ensure complete removal of the solvent. The fibers were cross-linked using $1 \%$ glutaraldehyde (Sigma-Aldrich, USA) solution for 24-h, rinsed by dipping in ethanol (20 times) for drying and then stored for different treatment conditions.

\subsection{Characterization of Composite Fabrics}

The scaffolds were fixed with $2.5 \%$ glutaraldehyde solution for 2 -h, dehydrated with series of ethanol from $50 \%$ to $100 \%$, then the fixed scaffolds were inserted onto aluminum, sputter with gold coating and then the morphological features of fabricated scaffolds were analyzed by using a scanning electron microscope (S4500; Hitachi, Tokyo, Japan). For structure analysis of the synthesized hydroxyapatite, the prepared scaffolds were mounted on a sample holder of $\mathrm{x}$-ray powder diffractometer (XRD; Rigaku Corporation, The Woodlands, TX, USA) under $\mathrm{Cu} \mathrm{K \alpha I}(\lambda=0.15406 \mathrm{~nm})$ radiation with $\mathrm{Ni}$ filter at $30 \mathrm{kV}$ potential and $15 \mathrm{~mA}$ current. At a speed $2 \% \mathrm{~min}$, the specimen was scanned in a range from $3^{\circ}$ to $50^{\circ}$. By using Jade 6.0 software (Materials Data Inc., Livermore, CA, USA), the obtained patterns were analyzed with model automated to the international center for diffraction (ICDD) database.

The dipole moment of molecule is changed under infrared excitation and different types of vibration at specific frequencies can be produced by different molecules. The fouriertransform infrared spectroscopy (FTIR) can use this characteristic to analyze the different functional groups of organic compounds. The composite fabrics analyzed in this study were ground and mixed thoroughly with potassium bromide, and then analyzed by using FTIR with the IR spectra analyzed set at a range of 450 to $4000 \mathrm{~cm}^{-1}$.

4.3. Harvest and Isolation of the Bone Marrow Stem Cells 
All animal experiments and surgical procedures were approved and carried out in compliance with the National Taiwan University, College of Medicine, Institutional Animal Care and Use Committee (IACUC) (no. 20180364).

Bone marrow stem cell (BMSC) was obtained from rats' femur as previously described [40]. Briefly, animals were anestheszed with intraperitoneal injection of a mixture of 1:2 concentration of Zoletil (Virbac AH, Inc., Fort Worth, TX, USA) and Rompum (Bayer, Taipei, Taiwan) ( $1 \mathrm{~mL} / \mathrm{kg}$ ), then sacrificed by rapid neck dislocation. The femur and tibiae bones were harvested and stripped all soft tissue. Under sterile condition, the bone marrow canals of femur and tibiae were flushed with Dulbecco's modified essential medium (DMEM, Life Technologies Inc., Gibco/Brl Division, Grand Island, New York, USA) to collect the bone marrow cells. The obtained cells were seeded in a $25 \mathrm{~cm}^{2}$ tissue culture flask and then incubated in DMEM supplemented with penicillin (100 UI/ml, Sigma-Aldrich, USA), streptomycin (100 $\mu \mathrm{g} / \mathrm{ml}$, Sigma-Aldrich, USA) and 10\% fetal bovine serum (FBS, Biological Industries, Israel) (at $37^{\circ} \mathrm{C}, 5 \% \mathrm{CO}_{2}$ ). At 24-h after seeding, the medium was repaced and the non-adherent cells were removed; then the culture medium was changed twice a week until the cells reached confluence (around 10-15 days).

4.4. Assessment of Cells Viability and Adhesion

Scaffold samples were tested with dot blotter apparatus for the effect on cellular viability on a 96-well plate. Briefly, strips of the electro-spun scaffolds were placed on the holes at the bottom of the dot blotter over a piece of parafilm (Parafilm M® All-Purpose Laboratory Film/ Bemis, Bio Life Haus, Malaysia) in the desired locations. Then, the blotter was sealed to hold electro-spun scaffolds in place. For the sterilization, the scaffolds were rinsed with $70 \%$ ethanol (5 min), washed with PBS (3 times) and irradiated with UV (254 $\mathrm{nm}$; for $30 \mathrm{~min}$ ). In this study, the BMSC cells were treated with the medium extracts of the negative control (aluminum oxide, $\mathrm{Al}_{2} \mathrm{O}_{3}$; Sigma-Aldrich, USA), positive control (zinc diethyldithiocarbamate, ZDEC; Sigma-Aldrich, USA), and the metformin-incorporated nano-gelatin/hydroxyapatite fibers (NGF), respectively, at the concentration of $0.2 \mathrm{~g} / \mathrm{mL}$ (at $37^{\circ} \mathrm{C}, 24$ hours).

The cells (BMSC: $5 \times 10^{3}$ cells/well) were then seeded and allowed to grow for 3 days, WST-1 assay was used to analyze cell viability and proliferation.

Briefly, $100 \mu \mathrm{L}$ of the selected medium was added to each well along with $10 \mu \mathrm{L}$ of WST1 reagent (Sigma-Aldrich, USA), incubated at $37^{\circ} \mathrm{C}$ for 1 -h. For background control, 100 $\mu \mathrm{L}$ of medium plus $10 \mu \mathrm{L}$ of WST-1 reagent was placed in a sterile 96-well plate. The medium with the WST-1 reagent was removed from each well and placed in the 96-well plate, then absorbance at $450 \mathrm{~nm}$ with a reference filter at $600 \mathrm{~nm}$ was read with a spectrophotometric plate reader (Thermo Fisher Automated ELISA Reader Multiskan ${ }^{\mathrm{TM}}$ FC Microplate Photometer, Thermo Fisher Scientific Inc., USA) to determine the amount of Formazan formation. The cell viability of control group (non-treated) was defined as $100 \%$.

For cells adhesion, the BMSCs $\left(1 \times 10^{5}\right.$ cells $\left./ \mathrm{mL}\right)$ seeded scaffolds 24 -well culture plate was incubated 4 -h (at $37{ }^{\circ} \mathrm{C}$ with $5 \% \mathrm{CO}_{2}$ ) and cultured for one month. At the end of 1month culture, the media was removed, the scaffold was treated with $4 \%$ buffered paraformaldehyde for $30 \mathrm{~min}$, permeabilized with 0.1\% Triton X-100 (Sigma-Aldrich, USA), and then incubated with Invitrogen Alexa Fluor ${ }^{\circledR} 488$ Phalloidin (Invitrogen/Thermo Fisher Scientific Inc., USA) for $30 \mathrm{~min}$ to stain the cytoskeleton protein. After washing with PBS, the nuclei were stained with 406-diamidino-2-phenylindole (DAPI, Invitrogen/Thermo Fisher Scientific Inc., USA) for $1 \mathrm{~min}$ and then observed with Nikon ECLIPSE Ti-s fluorescence microscope (Nikon, Japan).

4.5. Osteogenic Gene Expression Analysis

For cell differentiation and gene expression, the osteogenic medium used was DMEM supplemented with penicillin (100 UI/ml, Sigma-Aldrich, USA), streptomycin $(100 \mu \mathrm{g} / \mathrm{ml}$, Sigma-Aldrich, USA), 10\% fetal bovine serum (FBS, Biological Industries, Israel), $50 \mathrm{nM}$ 
dexamethasone (Sigma-Aldrich, USA), $0.2 \mathrm{mM}$ ascorbic acid (Sigma-Aldrich, USA), and $10 \mathrm{mM} \beta$-glycerophosphate (Sigma-Aldrich, USA). Quantitative real time polymerase chain reaction (qRT-PCR) assays were used to investigate the level of gene expression of alkaline phosphatase (ALP), runt-related transcription factor-2 (RUNX-2), osterix (SP7), osteocalcin (BGLAP), osteonectin (SPARC) and type I collagen (COL1A1). By using an RNA purification kit (ServicebioTechnology, Wuhan, China), total RNA was extracted, purity and concentration of the extracted RNAs were identified by Nanodrop 2000 (Thermo Fisher Scientific Inc., USA), then the diluted RNA was utilized for reverse transcription to generate cDNA. Finally, the obtained cDNA together with qPCR Mix (Tools Co., Taichung, Taiwan), primers (Tools Co., Taichung, Taiwan) and $\mathrm{dd}_{2} \mathrm{O}$ were put into qRT-PCR system (CFX Connect Real-Time PCR Detection System, BioRad, USA). The expression levels of different samples were normalized by GAPDH. Table 1 listed the PCR primers for quantitative real time polymerase chain reaction (qRT-PCR) used in this study. Transcription levels were normalized to GAPDH.

Table 1

The PCR primers for quantitative real time polymerase chain reaction (qRT-PCR).

\begin{tabular}{ll} 
Gene & Primer seqence \\
\hline ALP & Forward: 5'- GAGAAGCCGGGACACAGTTC -3' \\
RUNX2 & Reverse: 5'- CCTCCTCAACTGGGATGATGC-3' \\
& Forward: 5'- TAGGCGCATTTCAGGTGCTT-3' \\
SP7 & Reverse: 5'- GGTGTGGTAGTGAGTGGTGG-3 \\
& Forward: 5'-TAGGACTGTAGGACCGGAGC-3' \\
BGLAP & Reverse: 5'- CATAGTGAACTTCCTCCTGGGG-3' \\
& Forward: 5'-CTCACACTCCTCGCCCTATTG-3' \\
SPARC & Reverse: 5'- GCTTGGACACAAAGGCTGCAC-3' \\
& Forward: 5'- ATTGACGGGTACCTCTCCCA-3' \\
COL1a1 & Reverse: 5'-GAAAAAGCGGGTGGTGCAAT-3' \\
& Forward: 5'-AGAGGTCGCCCTGGAGC-3' \\
GAPDH & Reverse: 5'-CAGGAACACCCTGTTCACCA-3' \\
& Forward: 5'-AATGGGCAGCCGTTAGGAAA-3' \\
& Rreverse 5'-GCCCAATACGACCAAATCAGAG-3' \\
\hline
\end{tabular}

alkaline phosphatase: ALP, runt-related transcription factor-2: RUNX-2, osterix: SP7, osteocalcin: BGLAP, osteonectin: SPARC, collagen type I: COL1a1, glyceraldehyde-3phosphate dehydrogenase: GAPDH.

\subsection{Quantitation of Osteogenic Protein}

ELISA kits, including type I collagen (COL), alkaline phosphatase (ALP), osteonectin $(\mathrm{ON})$ and osteocalcin $(\mathrm{OC})(\mathrm{Abcam}, \mathrm{UK})$, were used for osteogenic protein analysis. Cells were co-cultured with scaffolds for four weeks. At every week, $100 \mu \mathrm{L}$ suspension or standard (Tools Co., Taichung, Taiwan) was drawn to each well, incubated for 1-h at $37^{\circ} \mathrm{C}$ and aspirated; then $100 \mu \mathrm{L}$ prepared Detection Reagent A was added, followed by 1 -h incubation at $37^{\circ} \mathrm{C}$. After that, wells were aspirated and washed 3 times. $100 \mu \mathrm{L}$ of prepared Detection Reagent B was added and incubated for 1 -h at $37^{\circ} \mathrm{C}$. Wells were aspirated and washed 5 times, then a $90 \mu \mathrm{L}$ TMB $\left(3,3^{\prime}, 5,5^{\prime}\right.$-Tetramethylbenzidine or TMB) Substrate was added and incubated for $1-\mathrm{h}$ at $37^{\circ} \mathrm{C}$. Finally, a $50 \mu \mathrm{L}$ Stop Solution was added and samples were read at 450nm immediately.

4.7. In Vivo Animal Study

A total of 27 rats were anesthetized with a 1:2 concentration of Zoletil (Virbac AH, Inc., Fort Worth, TX, USA) and Rompum (Bayer, Taipei, Taiwan) $(1 \mathrm{~mL} / \mathrm{kg})$ via intraperitoneal injection. Bilateral forelimbs of each animal were aseptically prepared. Skin incision was 
made to expose the ulnar bone, a 5-mm segmental bone defects were created in the midportion of the ulnar shaft by $5-\mathrm{mm}$ wide bone cutter (Rongeur) as the critical-sized bone defect. All bone debris in the defect site were washed out and clean away. The radius and ulna of rats are fused together by interosseous membrane, adequate stability can be achieved by leaving the radius intact without any fixation. Total number of 54 ulnar bone defects were created in all 27 rats. The 5-mm ulnar bone defects were then filled with either the metformin-incorporated nano-gelatin, hydroxyapatite fibrils (NGF) (NGF group), porous hydroxyapatite granules ("PURZER" SINBONE BONE REPLACEMENT, PURZER, Taipei, Taiwan: Sinbone group) or left alone without any filling material as a control; nine rats were used per group.

\subsection{Clinical Examination}

The post-surgical physical activities, surgical wound conditions (such as edema, hyperemia at the defect area, pain on palpation), and the appetite status of rats were observed. At 4, 8, and 12 post-surgical weeks, the rats were anesthetized with a 1:2 concentration of Zoletil (Virbac AH, Inc., Fort Worth, TX, USA) and Rompum (Bayer, Taipei, Taiwan) $(1 \mathrm{~mL} / \mathrm{kg})$ via intraperitoneal injection; then sacrificed by intracardiac injection of 150 $\mathrm{mg} / \mathrm{kg}$ potassium chloride (Taiwan Biotec Co., Ltd., Taoyuan, Taiwan). The animals were then quantitatively evaluated by using a high-resolution micro-computed tomography $(\mu-C T)$ (Skyscan1076, Kontich, Belgium). A region of interest (ROI: $5 \times 2 \times 2 \mathrm{~mm}$ ) around each defect was accurately defined and positioned for quantitative analysis. Then, 3D analysis of bone regeneration and quantification of bone defect density from the reconstructed ROI images was performed by CTAnSkyscan software.

4.9. Histological Analysis

After $\mu$-CT scanning, the specimens were fixed and decalcified by $20 \%$ EDTA (adjusted to 7.4 with $\mathrm{NaOH}$; Sigma-Aldrich, USA) in a refrigerator at $4^{\circ} \mathrm{C}$ with intermittent shaking to make sure the decalcifying solution was flowing around the bone. The EDTA solution was changed twice or three times a week till the bone was soft and pliable (usually 2 weeks). The specimens were rinsed with distilled $\mathrm{H}_{2} \mathrm{O}$ for 3 times, embedded in paraffin, sectioned at a thickness of 5- $\mu \mathrm{m}$ and mounted on glass slide, then stained with hematoxylin and eosin (H\&E) or Goldner's Trichrome staining, and then examined under a light microscope (IX71; Olympus, Tokyo, Japan). The newly formed bone tissue was visualized by using MetaMorph Microscopy Automation and Image Analysis Software (Molecular Devices, LLC. San Jose, CA, USA).

\subsection{Statistical Analysis}

All data were expressed as mean \pm standard deviation (SD). Statistical analysis was performed by using one-way ANOVA and the post hoc comparisons used was Bonferroni test. Statistically significance was defined at p-value less than 0.05 . All analyses were performed by using SPSS version 16.0 software (SPSS Inc., Chicago, USA).

\section{Conclusions}

In summary, we demonstrated that the metformin-incorporated nano-gelatin-hydroxyapatite scaffold improved healing potential of pure gelatin or HA scaffold. Although metformin-incorporated nano-gelatin-hydroxyapatite scaffolds had advantageous effectiveness during critical-sized bone defect regeneration, further validation is required before it can be applied to clinical applications.

Supplementary Materials: The following are available online at www.mdpi.com/xxx/s1, Figure S1: title, Table S1: title, Video S1: title.

\section{Materials and methods}

Surgical procedure 
We used 2 skeletally mature female Lam Yu-100 mini pigs (aged 10-14 months, weight 40-50 kg). Those were initially assigned into two groups based on the amount of metformin-incorporated nano-gelatin/ hydroxyapatite fibrous scaffold placed per side (cage insertion after C5 vertebral body resection; proximal end with, distal end without scaffold). This research protocol was approved by the Institutional Animal Care and Use Committee (Affidavit of Approval of Animal Use Protocol, IACUC of Pigmodel Animal Technology Co., Ltd.: Approval No. PIG-108026).

Under general anesthesia with an intramuscular injection of ketamine and xylazine, a single-level total en bloc spondylectomy of C5 vertebral body was performed. The end plate cartilage of adjacent level were removed and the spinal fusion between C4 and C6 were performed after insertion of custom-made porous Titanium cage. We made an anterior lateral oblique skin incision in the neck region, followed by subperiosteal dissection of neck. We removed the disc material of C4-5 and C5-6 interspace and then total en bloc spondylectomy of the C5 vertebral body using rongeurs and an electric-driven burr (Stryker, Kalamazoo, MI, USA). Then, the graft materials were prepared as described later and implanted in proximal end of the custom-made implant and fixation was performed using 2 screws ( $3.5 \mathrm{~mm}$ diameter, 25-35 $\mathrm{mm}$ long). AThe fascial incision was closed with 2-0 absorbable sutures, and the skin was reapproximated with 2-0 nylon sutures. The animals were given intravenous antibiotics (cefazolin) during surgery and continued for 3 days. The mini pigs were allowed to move about their cages without any external immobilization. All animals were euthanized at 3 months after surgery by intravenous injection of an anesthetic and $\mathrm{KCl}$.

\section{Assessment of spinal fusion}

Three months after surgery, all animals were euthanized and the cervical vertebrae were carefully harvested. Spinal fusion was assessed by five different methods: manual palpation, plain radiography, three-dimensional (3D) computed tomography (CT).

\section{$\underline{\text { Results }}$}

A. Plain radiograph taken 3 months after total en bloc spondylectomy of C-5 vertebral body and cage implantation.

Four different levels chosen for comparison were selected: (a) proximal end of implanted cage; (b) $1 \mathrm{~cm}$ distal to level-a; (c) $1 \mathrm{~cm}$ proximal to level-d; (d) distal end of implanted cage.

B. CT scan of $\mathrm{C}$ spine taken 3 months after surgery.

C. Enlarged film of B showed good bony growth at the proximal end (level a: with NGF) of C5 cage in contrast to the poor bone incorporation of distal end (level-d: without NGF).

D. Section of different part of ROI showed good bony regeneration and incorporation of new bone with upper end of C5 cage (D-a); while poor bony growth and incorporation with C5 cage at the lower end (D-d). 
A

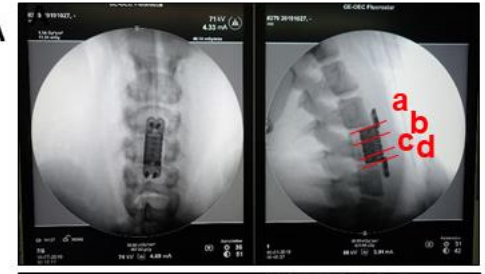

B

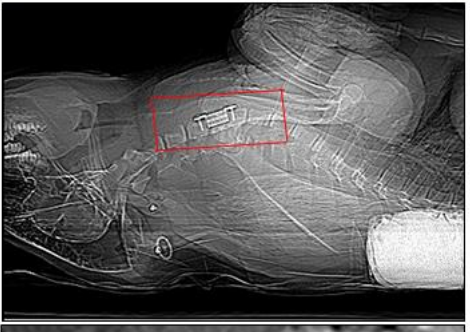

C

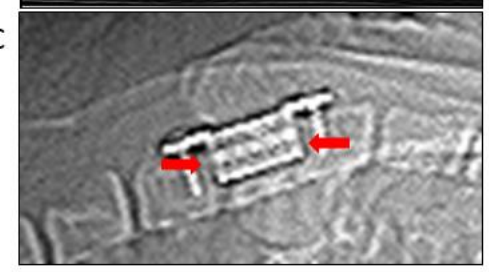

D-a

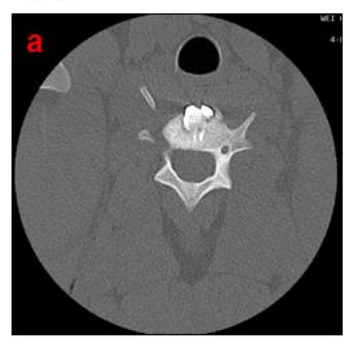

\section{D-c}

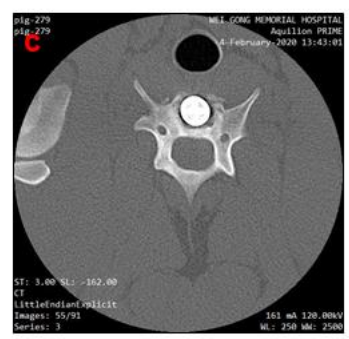

D-b

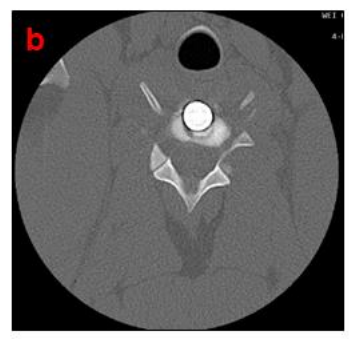

D-d

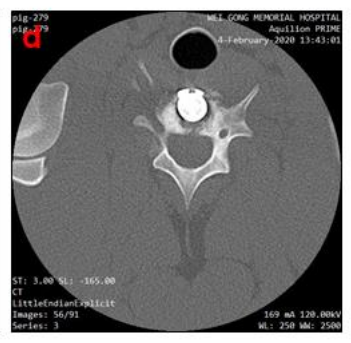

Fig. Supplementary Preliminary results of LanYu-100 minipig model.

A. Plain radiograph taken 3 months after total en bloc spondylectomy of C-5 vertebral body and cage implantation. Four different levels chosen for comparison were selected: (a) proximal end of implanted cage; (b) $1 \mathrm{~cm}$ distal to level-a; (c) $1 \mathrm{~cm}$ proximal to level-d; (d) distal end of implanted cage. B. CT scan of C spine taken 3 months after surgery. C. Enlarged film of B showed good bony growth at the proximal end (level a: with NGF) of C5 cage in contrast to the poor bone incorporation of distal end (level-d: without NGF). D. Section of different part of ROI showed good bony regeneration and incorporation of new bone with upper end of $\mathrm{C} 5$ cage (D-a); while poor bony growth and incorporation with C5 cage at the lower end (D-d).

Author Contributions: PW Weng: Investigation, Validation, Formal analysis, Writing - review \& editing. CK Sun: Investigation, Validation, Formal analysis, Writing - original draft, review \& editing. FY Tsuang: Investigation, Writing - review \& editing. YW Lin: Investigation, Writing - review \& editing. JS Sun: Conceptualization, Supervision, Project administration, Funding acquisition, Investigation, Validation, Formal analysis, Writing - review \& editing. FH Lin: Conceptualization, Supervision, Project administration, Writing - original draft, review \& editing. TH Tsai: Conceptualization, Supervision, Project administration, Writing - review \& editing. ZC Chang: Conceptualization, Supervision, Project administration, Investigation, Validation, Writing - review \& editing. All authors have read and approved the final submitted manuscript.

Funding: This work was supported by the Institute of Biomedical Engineering, National Taiwan University, Taipei, Taiwan, ROC, Ministry of Science and Technology, Taipei, Taiwan, ROC [MOST 108-2314-B-002-120-MY3] and the National Taiwan University Hospital, Taipei, Taiwan, ROC [UN108-002].

\section{Institutional Review Board Statement: Not applicable.}

Ethics approval and consent to participate: All animal procedures and experiments were performed following a protocol approved by the National Taiwan University, College of Medicine and Public Health, Institutional Animal Care and Use Committee (IACUC no. 20180364).

Data Availability Statement: The datasets used and/or analyzed in the current study are available from the corresponding author on reasonable request. 


\begin{abstract}
Acknowledgments: The authors would like to express their immense gratitude to the Institute of Biomedical Engineering, National Taiwan University, Ministry of Science and Technology and the National Taiwan University Hospital for their financial support. We thank the staff of the Second Core Lab, Department of Medical Research, National Taiwan University Hospital for technical support during the study. We also thank the (Wizdom Inc., Las. Vegas, NV89148, USA) and Ingrowth biotech (Kaohsiung, Taiwan 821) for their kindness of providing the prescription or recipe of their product.
\end{abstract}

Conflicts of Interest: The authors declare that they have no competing interests.

\title{
References
}

1. 1. Taichman RS. Blood and bone: two tissues whose fates are intertwined to create the hematopoietic stem-cell niche. Blood. 2005;105(7):2631-9.

2. 2. Yi S, Ding F, Gong L, Gu X. Extracellular Matrix Scaffolds for Tissue Engineering and Regenerative Medicine. Current stem cell research \& therapy. 2017;12(3):233-46.

3. 3. Brodsky B, Persikov AV. Molecular structure of the collagen triple helix. Advances in protein chemistry. 2005;70:301-39.

4. 4. Clarke B. Normal bone anatomy and physiology. Clinical journal of the American Society of Nephrology : CJASN. 2008;3 Suppl 3:S131-9.

5. 5. Landis WJ. The strength of a calcified tissue depends in part on the molecular structure and organization of its constituent mineral crystals in their organic matrix. Bone. 1995;16(5):533-44.

6. 6. Echave MC, Saenz del Burgo L, Pedraz JL, Orive G. Gelatin as Biomaterial for Tissue Engineering. Current pharmaceutical design. 2017;23(24):3567-84.

7. 7. Ranganathan S, Balagangadharan K, Selvamurugan N. Chitosan and gelatin-based electrospun fibers for bone tissue engineering. Int J Biol Macromol. 2019;133:354-64.

8. 8. Massoumi B, Abbasian M, Jahanban-Esfahlan R, Mohammad-Rezaei R, Khalilzadeh B, Samadian H, et al. A novel bioinspired conductive, biocompatible, and adhesive terpolymer based on polyaniline, polydopamine, and polylactide as scaffolding biomaterial for tissue engineering application. Int J Biol Macromol. 2020;147:1174-84.

9. 9. Lotfi G, Shokrgozar MA, Mofid R, Abbas FM, Ghanavati F, Baghban AA, et al. Biological Evaluation (In Vitro and In Vivo) of Bilayered Collagenous Coated (Nano Electrospun and Solid Wall) Chitosan Membrane for Periodontal Guided Bone Regeneration. Ann Biomed Eng. 2016;44(7):2132-44.

10. 10. Kim BR, Nguyen TB, Min YK, Lee BT. In vitro and in vivo studies of BMP-2-loaded PCL-gelatin-BCP electrospun scaffolds. Tissue Eng Part A. 2014;20(23-24):3279-89.

11. 11. Zhao X, Zhou L, Li Q, Zou Q, Du C. Biomimetic mineralization of carboxymethyl chitosan nanofibers with improved osteogenic activity in vitro and in vivo. Carbohydr Polym. 2018;195:225-34.

12. 12. Zhang X, Meng S, Huang Y, Xu M, He Y, Lin H, et al. Electrospun Gelatin/beta-TCP Composite Nanofibers Enhance Osteogenic Differentiation of BMSCs and In Vivo Bone Formation by Activating Ca (2+) -Sensing Receptor Signaling. Stem Cells Int. 2015;2015:507154.

13. 13. Klotz BJ, Gawlitta D, Rosenberg A, Malda J, Melchels FPW. Gelatin-Methacryloyl Hydrogels: Towards BiofabricationBased Tissue Repair. Trends Biotechnol. 2016;34(5):394-407.

14. 14. Driscoll JA, Lubbe R, Jakus A, Chang K, Haleem M, Yun C, et al. 3D-printed Ceramic-Demineralized Bone Matrix Hyperelastic Bone Composite Scaffolds for Spinal Fusion. Tissue Eng Part A. 2019.

15. 15. Frohbergh ME, Katsman A, Mondrinos MJ, Stabler CT, Hankenson KD, Oristaglio JT, et al. Osseointegrative properties of electrospun hydroxyapatite-containing nanofibrous chitosan scaffolds. Tissue Eng Part A. 2015;21(5-6):970-81.

16. 16. Molinuevo MS, Schurman L, McCarthy AD, Cortizo AM, Tolosa MJ, Gangoiti MV, et al. Effect of metformin on bone marrow progenitor cell differentiation: in vivo and in vitro studies. J Bone Miner Res. 2010;25(2):211-21.

17. 17. Oryan A, Alidadi S, Bigham-Sadegh A, Moshiri A. Comparative study on the role of gelatin, chitosan and their combination as tissue engineered scaffolds on healing and regeneration of critical sized bone defects: an in vivo study. J Mater Sci Mater Med. 2016;27(10):155.

18. 18. Parizi AM, Oryan A, Shafiei-Sarvestani Z, Bigham AS. Human platelet rich plasma plus Persian Gulf coral effects on experimental bone healing in rabbit model: radiological, histological, macroscopical and biomechanical evaluation. J Mater Sci Mater Med. 2012;23(2):473-83.

19. 19. Yazdimamaghani M, Vashaee D, Assefa S, Walker KJ, Madihally SV, Kohler GA, et al. Hybrid macroporous gelatin/bioactive-glass/nanosilver scaffolds with controlled degradation behavior and antimicrobial activity for bone tissue engineering. J Biomed Nanotechnol. 2014;10(6):911-31.

20. 20. Takahashi Y, Yamamoto M, Tabata Y. Enhanced osteoinduction by controlled release of bone morphogenetic protein-2 from biodegradable sponge composed of gelatin and beta-tricalcium phosphate. Biomaterials. 2005;26(23):4856-65.

21. 21. Kerney R, Gross JB, Hanken J. Runx2 is essential for larval hyobranchial cartilage formation in Xenopus laevis. Dev Dyn. 2007;236(6):1650-62. 
22. 22. Zheng C, Wang J, Liu Y, Yu Q, Liu Y, Deng N, et al. Functional Selenium Nanoparticles Enhanced Stem Cell Osteoblastic Differentiation through BMP Signaling Pathways. Advanced Functional Materials. 2014;24(43):6872-83.

23. 23. Kaur G, Valarmathi MT, Potts JD, Wang Q. The promotion of osteoblastic differentiation of rat bone marrow stromal cells by a polyvalent plant mosaic virus. Biomaterials. 2008;29(30):4074-81.

24. 24. Lu T, Liu X, Qian S, Cao H, Qiao Y, Mei Y, et al. Multilevel surface engineering of nanostructured TiO2 on carbon-fiberreinforced polyetheretherketone. Biomaterials. 2014;35(22):5731-40.

25. 25. Huang Z, Nelson ER, Smith RL, Goodman SB. The sequential expression profiles of growth factors from osteoprogenitors [correction of osteroprogenitors] to osteoblasts in vitro. Tissue Eng. 2007;13(9):2311-20.

26. 26. Aubin JE. Regulation of osteoblast formation and function. Rev Endocr Metab Disord. 2001;2(1):81-94.

27. 27. Quarles LD, Yohay DA, Lever LW, Caton R, Wenstrup RJ. Distinct proliferative and differentiated stages of murine MC3T3-E1 cells in culture: an in vitro model of osteoblast development. J Bone Miner Res. 1992;7(6):683-92.

28. 28. Hoemann CD, El-Gabalawy H, McKee MD. In vitro osteogenesis assays: influence of the primary cell source on alkaline phosphatase activity and mineralization. Pathol Biol (Paris). 2009;57(4):318-23.

29. 29. Klein C, de Groot K, Chen W, Li Y, Zhang X. Osseous substance formation induced in porous calcium phosphate ceramics in soft tissues. Biomaterials. 1994;15(1):31-4.

30. 30. Yamasaki H, Sakai H. Osteogenic response to porous hydroxyapatite ceramics under the skin of dogs. Biomaterials. 1992;13(5):308-12.

31. 31. Zhang K, Zhou Y, Xiao C, Zhao W, Wu H, Tang J, et al. Application of hydroxyapatite nanoparticles in tumor-associated bone segmental defect. Sci Adv. 2019;5(8):eaax6946.

32. 32. Ozturk A, Yetkin H, Memis L, Cila E, Bolukbasi S, Gemalmaz HC. Demineralized bone matrix and hydroxyapatite/tricalcium phosphate mixture for bone healing in rats. Int Orthop. 2006;30(3):147-52.

33. 33. Khan MN, Islam JM, Khan MA. Fabrication and characterization of gelatin-based biocompatible porous composite scaffold for bone tissue engineering. J Biomed Mater Res A. 2012;100(11):3020-8.

34. 34. Gholipour H, Meimandi-Parizi A, Oryan A, Bigham Sadegh A. The effects of gelatin, fibrin-platelet glue and their combination on healing of the experimental critical bone defect in a rat model: radiological, histological, scanning ultrastructural and biomechanical evaluation. Cell Tissue Bank. 2018;19(3):341-56.

35. 35. Szalaj U, Swiderska-Sroda A, Chodara A, Gierlotka S, Lojkowski W. Nanoparticle Size Effect on Water Vapour Adsorption by Hydroxyapatite. Nanomaterials (Basel). 2019;9(7).

36. 36. Rios-Pimentel FF, Chang R, Webster TJ, Mendez-Gonzalez MM, Garcia-Rocha M. Greater osteoblast densities due to the addition of amphiphilic peptide nanoparticles to nano hydroxyapatite coatings. Int J Nanomedicine. 2019;14:3265-72.

37. 37. Wang C, Li H, Chen SG, He JW, Sheng CJ, Cheng XY, et al. The skeletal effects of thiazolidinedione and metformin on insulin-resistant mice. J Bone Miner Metab. 2012;30(6):630-7.

38. 38. Wang YG, Qu XH, Yang Y, Han XG, Wang L, Qiao H, et al. AMPK promotes osteogenesis and inhibits adipogenesis through AMPK-Gfi1-OPN axis. Cell Signal. 2016;28(9):1270-82.

39. 39. Kanazawa I, Takeno A, Tanaka KI, Notsu M, Sugimoto T. Osteoblast AMP-activated protein kinase regulates glucose metabolism and bone mass in adult mice. Biochem Biophys Res Commun. 2018;503(3):1955-61.

40. Jiating L, Buyun J, Yinchang Z. Role of Metformin on Osteoblast Differentiation in Type 2 Diabetes. Biomed Res Int. 2019;2019:9203934. 